\title{
Behavior of HAWT with Differential Planetary Gearbox
}

\author{
Marat Z. Dosaev1, Liubov A. Klimina1, Yury D. Selyutskiy', Mi-Ching Tsai², Hong-Tzer Yang ${ }^{3}$ \\ ${ }^{1}$ Institute of Mechanics, Lomonosov Moscow State University, Moscow, Russia \\ ${ }^{2}$ Department of Mechanical Engineering, National Cheng Kung University, Tainan City, Taiwan \\ ${ }^{3}$ Department of Electrical Engineering, National Cheng Kung University, Tainan City, Taiwan \\ Email: dosayev@imec.msu.ru
}

Received December 2013

\begin{abstract}
A dynamic model for simulating behavior of a horizontal axis wind turbine (HAWT) with differential planetary gearbox is developed. The aerodynamic load applied to the wind turbine connected with the carrier is described using the quasi-steady approach. The control torque is assumed to be applied to the external ring of the gearbox. Steady regimes of the device are analyzed, and their stability is studied. For the case of constant control torque, power costs are estimated required for preserving constant angular speed of the generator.
\end{abstract}

Keywords

HAWT; Wind Engineering; Differential Planetary Gearbox; Stability

\section{Introduction}

Wind power systems in the world power industry gain more and more importance for many reasons, ranging from ecological to political. A wide scope of engineering and scientific research of wind turbine electrodynamics is reflected in numerous papers and patents ([1]).

Practical tasks in wind turbine applications often impose requirements on an angular speed of a rotor of a generator. In particular, a very common requirement is to maintain angular speed of the rotor within a certain range near a specific value. For this purpose, overdrive transmission gears are used, the most wide-spread of which is a planet gear. One of more advanced types of such devices is a differential planet gear (DPG) that allows controlling the output angular speed. This mechanism consists of two coaxial gearwheels with different diameters (an external ring and a so-called sun), several small gearwheels (so-called planets), and a carrier to which centers of planets are connected.

Planet gears are widely used in engineering (e.g., in automotive industry). In the same time, introduction of this mechanism into wind turbine construction can be considered as a rather new approach revealing a variety of innovative opportunities. In particular, DPG can be used for control of a wind turbine dynamics under changing external conditions of operation (for instance, variable wind speed or resistance of the external circuit).

In this paper, the wind power system with DPG is studied, and possibilities for its control are analyzed. The mechanical system under consideration consists of a horizontal axis wind turbine (HAWT), a DPG and a gene- 
rator (Figure 1). Axis of the turbine is connected to the carrier of the gear box. Rotor of the generator is connected to the sun gear, the generator being connected to the electrical circuit with changeable external resistance.

Rotation of the external ring of the differential planetary gear box is supposed to be controlled via external control torque applied to this ring. In contrast to many other investigations of DPG applications for wind power systems (e.g., [1-5]) this paper deals with dynamics of the so-called small-scale stand-alone wind power system (not connected to the grid).

Such systems can be used for remote households and infrastructural objects, for camping trips and expeditions. Distinguishing feature of dynamics of an autonomous power system is that the generator of such system represents the only electrical power source in its circuit. In this work it is taken into account that electromechanical torque acting on the generator rotor depends on external resistance in the circuit of the generator as well as on electromechanical properties of the generator.

\section{Problem Statement and Motion Equations}

Assume that the HAWT is located in a wind flow with the speed $V$. All elements of DPG are rigid bodies, and there is no slipping between them. Axis of the turbine is rigidly connected to the carrier of DPG. Let $\omega_{c}$ be the angular speed of the turbine. The angular speed of the generator rotor is equal to the angular speed $\omega_{s}$ of the sun gear. Angular speed of the external ring is $\omega_{r}$.

External torques acting on the system are as follows: an aerodynamic torque $T_{c}$ acting on the turbine (and thereby to the carrier), electromechanical torque $T_{s}$ acting on the generator rotor (and, hence, onto the sun gear) due to consumers present in the external circuit of the generator, and the control torque $T_{r}$ applied he external ring of the DPG.

The following constant parameters of the model are introduced: $r_{c}, r_{s}, r_{p}, r_{r}$ are radiuses of the carrier, sun gear, planet gear and external ring respectively, such that

$$
r_{c}=r_{s}+r_{p}, r_{r}=r_{c}+r_{p}
$$

Supposing that there is no slipping in the DPG, we obtain the following kinematical relations:

$$
\omega_{p} r_{p}=\omega_{r} r_{r}-\omega_{c} r_{c}, \omega_{s} r_{s}=2 \omega_{c} r_{c}-\omega_{r} r_{r}
$$

The aerodynamic torque is assumed to be determined with the following formula:

$$
T_{c}=\frac{1}{2} \rho S b V^{2} f(\lambda) .
$$

where $\lambda=b \omega_{c} / V$ is TSR, $b$ is turbine radius, $S$ is the characteristic area of the turbine.

The function $f(\lambda)$ (non-dimensional aerodynamic torque) was identified for a particular wind turbine basing on experiments performed in the Institute of Mechanics:

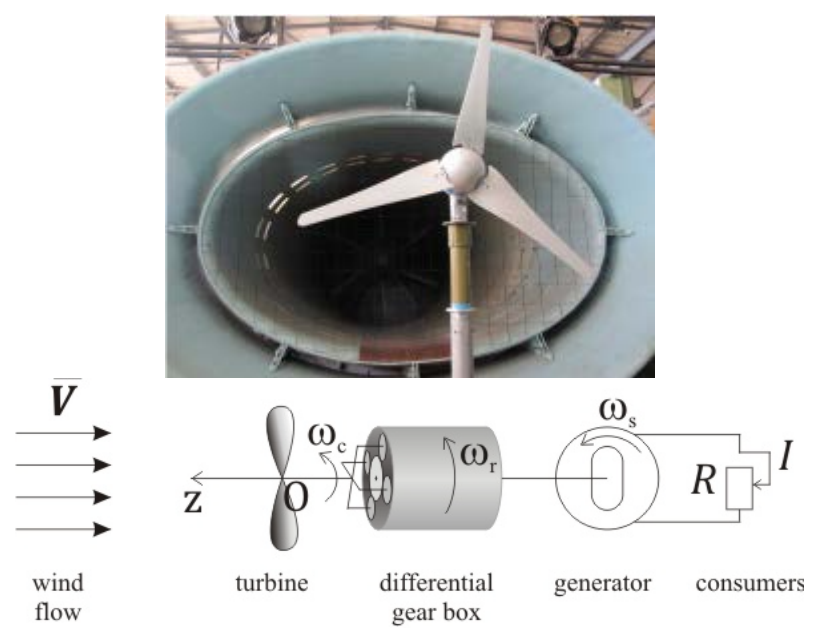

Figure 1. Experimental setup in the wind tunnel of the LMSU Institute of Mechanics and a scheme of the system. 


$$
f(\lambda)=-0.00016 \lambda^{5}+0.0047 \lambda^{4}-0.048 \lambda^{3}+0.19 \lambda^{2}-0.16 \lambda+0.11
$$

Results are shown in Figure 2, where dots denote experimental data and the solid line means approximation curve. Evidently, agreement is good enough.

External load upon the turbine and generator is modeled accordingly to the $[6,7]$. So the expression for electromechanical torque acting on the rotor of the generator is the following:

$$
T_{s}=c \omega_{s}
$$

Here $c$ is the coefficient of electromechanical interaction.

In what follows we assume that the inductance of the generator is small enough, so that the current $I$ in the external circuit is given by the following formula:

$$
I=c \omega_{s}(R+r)^{-1},
$$

where $R$ is resistance of the external circuit, and $r$ is internal resistance of the generator.

Motion equations of the considered system can be written in the following non-dimensional form:

$$
\begin{gathered}
\dot{\lambda}=A_{1} f(\lambda)-A_{2} u-A_{3} \mu . \\
\dot{\mu}=B_{1} f(\lambda)+B_{2} u-B_{3} \mu .
\end{gathered}
$$

Here $\tau=V t / b$ is non-dimensional time (dot denotes derivative with respect to $\tau$ ), $\mu=\mu_{0} \omega_{s}$ is normalized angular speed of the generator, $u=-U T_{r}$ is non-dimensional control ( $T_{r}$ is control torque applied to the ring), and $A_{1-3}, B_{1-3}, U, \mu_{0}$ are positive constants.

\section{Steady Regimes in Case of Constant Control}

Assume now that the control torque is constant: $u \equiv u_{0}$. Then, taking into account (1), one can readily show that the dynamic system (2)-(3) can have different number of fixed points for different values of $u_{0}$ : for small enough and large enough values there exists only one steady regime, while in the intermediate range there are three such regimes.

Stability condition of steady regimes looks as follows:

$$
f^{\prime}(\lambda)<0 \text {. }
$$

Note that if there exists only one steady solution, it is asymptotically stable. If there exist three such solutions, then those with the highest and the lowest angular speeds are asymptotically stable, and the one with the "medium" angular speed is unstable (this fixed point is saddle).

\section{Output Power Estimation}

The common task for a DPG mechanism is to maintain a preliminary chosen value of the angular speed of a sun gear even in changeable external conditions. This value depends on technical requirements of a particular generator.

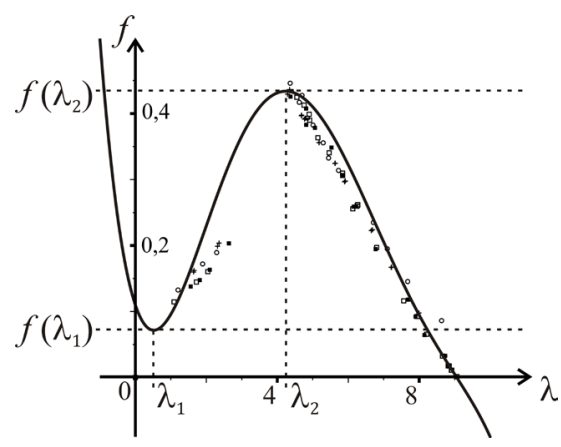

Figure 2. The identified function of normalized aerodynamic torque. 
For the case of a wind power system, such operation conditions as wind speed and external load change frequently. The control torque is applied to achieve a needed value of the angular speed of the sun. Power for supplying of the control mechanism can be taken from some storage-additional source of energy that is used to overcome short periods of unfriendly exploitation conditions without brakes in the system performance.

This storage can be replenished from the power produced by the wind power generator during advantage periods of exploitation conditions: i.e. while the wind is strong enough or while the number of primary consumers is small.

It is necessary to give some estimation of power costs required by the control system, in order to calculate what should be the profit of wind power system exploitation in the region with a given range of wind speed and for supposed values of external load from consumers that may change.

Assume that angular speed of the sun on an operation mode should be equal to $\omega_{s}^{*}$.

From equations of steady regimes we can obtain the required value of the control torque $T_{r}$ and estimate power costs for the control: mechanical power necessary for the control equals to $P_{\text {cost }}=T_{r} \omega_{r}$.

If the value $P_{\text {cost }}$ is less than zero, it means that control torque is used not to accelerate the external ring gear but to decelerate it, hence, in such case no power is spent for the control and even additional power can be trapped from the external ring rotation.

The output electrical power is represented by the value $P_{\text {out }}=I^{2} R$, where $I$ is the current in the external circuit, and $R$ is the external resistance.

Hence, in order to estimate the profit power, the following expression can be used:

$$
P_{\text {profit }}=I^{2} R-T_{r} \omega_{r} \text {. }
$$

On Figures 3-6, examples of power estimation for the system with given parameters are presented for two illustrative cases. In the first case (Figures 3 and 4$)$ it is assumed that external load doesn't change (100 Ohm) while wind speed $V$ can take any value from 0 to $15 \mathrm{mps}$. In the second case (Figures $\mathbf{5}$ and $\mathbf{6}$ ) calculations are performed for fixed wind speed (12 mps), but for different values of the external load.

For both cases the hysteretic behavior is obtained: the final profit power in a certain range depends on the direction of changing of external conditions (both wind speed and external resistance).

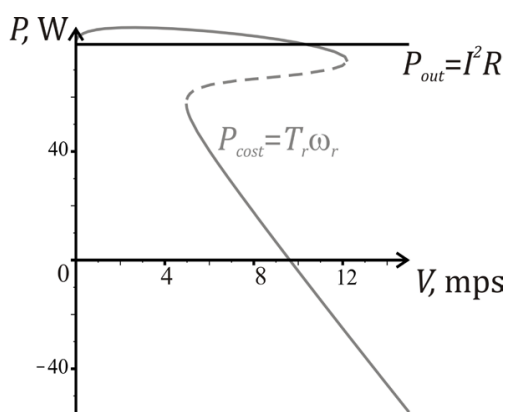

Figure 3. Estimation of the output power and costs for control for $R=100 \mathrm{Ohm}$.

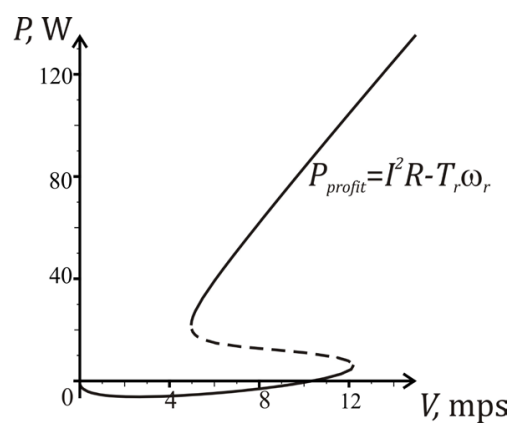

Figure 4. Estimation of the profit power for $R=100 \mathrm{Ohm}$. 


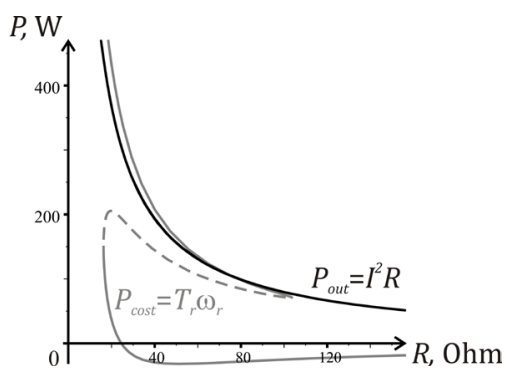

Figure 5. Estimation of the output power and costs for control for $V=12 \mathrm{mps}$.

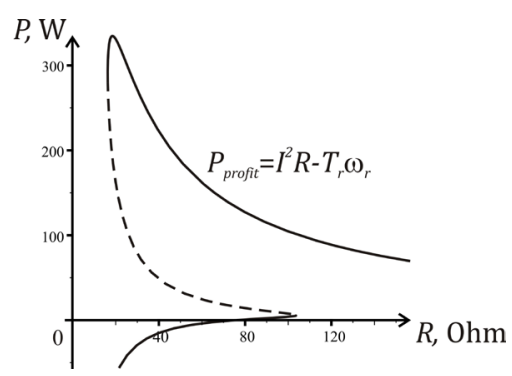

Figure 6. Estimation of the profit power for $V=12 \mathrm{mps}$.

\section{Conclusion}

The behavior of the wind power system including differential planet gear was analyzed. Conditions of stability of steady solutions of the corresponding dynamic system were obtained. Steady operation modes corresponding to stable steady solutions were studied depending on changing external conditions. Power costs for the control were estimated for the system with a certain set of model parameters.

\section{Acknowledgements}

This work was partially supported by the Russian Foundation for Basic Research, projects NN 14-08-01130, 11-08-92005, and 12-01-00364 and by the Taiwan National Science Council.

\section{References}

[1] Chen, Z., Guerrero, M. and Blaabjerg, F. (2009) A Review of the State of the Art of Power Electronics for Wind Turbines. IEEE Transactions on Power Electronics, 24, 1859-1875. http://dx.doi.org/10.1109/TPEL.2009.2017082

[2] Müller, H., Pöller, M., Basteck, A., Tilscher, M. and Pfister, J. (2006) Grid Compatibility of Variable Speed Wind Turbines with Directly Coupled Synchronous Generator and Hydro-Dynamically Controlled Gearbox. 6th Int'l Workshop on Large-Scale Integration of Wind Power and Transmission Networks for Offshore Wind Farms, Delft, 26-28 October 2006, 307-315.

[3] Polinder, H., van der Pijl, A.F.F., de Vilder, G.-J. and Tavner, P.J. (2006) Comparison of Direct-Drive and Geared Generator Concepts for Wind Turbines. IEEE Transactions on Energy Conversion, 21, 725-733. http://dx.doi.org/10.1109/TEC.2006.875476

[4] Rossi, C., Corbelli, P. and Grandi, G. (2009) W-CVT Continuously Variable Transmission for Wind Energy Conversion System. http://www.die.ing.unibo.it/pers/grandi/papers/PEMWA2009.pdf

[5] Tsai, M.-C. and Huang, C.-C. (2012) Development of a Variable-Inertia Device with a Magnetic Planetary Gear-Box. IEEE-ASME Transactions on Mechatronics, 16, 1120-1128. http://dx.doi.org/10.1109/TMECH.2010.2077679

[6] Dosaev, M.Z., Kobrin, A.I., Lokshin, B.Ya., Samsonov, V.A. and Selyutskiy, Yu.D. (2007) Constructive Theory of Small-Scale Wind Power Generators. Part I-II. MSU Publishing, Moscow.

[7] Dosaev, M.Z., Samsonov, V.A. and Seliutski, Yu.D. (2007) On the Dynamics of a Small-Scale wind Power Generator. Doklady Physics, 52, 50-53. http://dx.doi.org/10.1134/S1028335807090091 